International Journal of English Language Studies (IJELS)

ISSN: $2707-7578$

DOI: $10.32996 /$ ijels

Website: https://al-kindipublisher.com/index.php/ijels

\title{
A Corpus-Based Study on Conceptual Metaphors in the Finance \& Economics Column of The Economist
}

\author{
Songman Liang $\mathbf{8}(\mathrm{D})$ \\ Graduate student, School of English for International Business, Guangdong University of Foreign Studies, China \\ $\bowtie$ Corresponding Author: Songman Liang, E-mail: 2392898385@qq.com
}

\author{
ARTICLE INFORMATION \\ Received: July 06, 2021 \\ Accepted: August 05, 2021 \\ Volume: 3 \\ Issue: 8 \\ DOI: $10.32996 /$ ijels.2021.3.8.3
}

\section{KEYWORDS}

Conceptual metaphors; Economic discourse; The Economist; Finance \& Economics Column

\section{ABSTRACT}

Traditional metaphor researches consider metaphors as a rhetoric device for ornamental study. In 1980, Lakoff and Johnson put forward the Conceptual Metaphor Theory, which marks the shift of metaphor study from rhetoric view to cognitive view. Since then, numerous studies at home and abroad on conceptual metaphors have emerged. Economic news has also become a research interest. However, few research concerns about The Economist, let alone Finance \& Economics Column inside. Therefore, this study explores the conceptual metaphors in the Finance \& Economics Column of the Economist with Conceptual Metaphor Theory as a theoretical foundation. In order to address the above questions, the paper selects articles from October 2019 to December 2019 in The Economist and employs both qualitative and quantitative approaches to analyze conceptual metaphors in the self-constructed corpus. The results show that: firstly, altogether 443 conceptual metaphors are identified in the corpus, covering structural metaphor, ontological metaphor and orientational metaphor. Due to space limitation, only JOURNNEY metaphor, HUMAN BEING metaphor and UP/DOWN metaphor with high frequency is selected to be analyzed in detail. And their frequency varies from each other. Secondly, these three metaphors are identified in the corpus function by mapping from the source domain to the target domain. Finally, the frequency of these three metaphors is different lies in the systematicity, cultural coherence of metaphors and characteristics of economic news. This study enlarges the scope of conceptual metaphor and helps enhance their metaphorical awareness in economic discourses.

\section{Introduction}

Traditionally, metaphor is regarded as a rhetoric device for decorative use. The publication of Lakoff and Johnson's work Metaphors We Live By signifies the shift of metaphor study from the rhetorics view to the cognitive view. In their work, Lakoff and Johnson put forward Conceptual Metaphor Theory and claim that metaphor is ubiquitous in our daily life and our cognition is metaphorical in nature. Since then, numerous scholars have done research with conceptual metaphor as a theoretical framework and many studies on different types of discourses from the perspective of conceptual metaphor emerge, including economic news.

However, as the literature review indicates, few researchers explore conceptual metaphors in The Economist, let alone Finance \& Economics Column inside. The Economist is a kind of English journal widely acknowledged by both native speakers and nonnative speakers. The Economist is a financial journey, so it includes many abstract economic terminologies which are difficult for readers to understand. Besides, Conceptual metaphors allow people to understand abstract concepts in terms of concrete concepts.
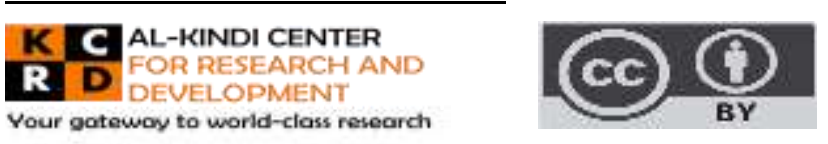

Published by Al-Kindi Center for Research and Development. Copyright (c) the author(s). This open access article is distributed under a Creative Commons Attribution (CC-BY) 4.0 license 
Therefore, this paper intends to explore the conceptual metaphors in the Finance \& Economics Column of The Economist. Specifically, it analyses the distribution of three types of conceptual metaphors and explores their mapping process. This paper has theoretical and practical significance. Theoretically, this paper expands the study scope of conceptual metaphor by studying it at the discourse level. Practically, this study contributes to readers' comprehension of abstract economic terminologies and enhances their metaphorical awareness in economic discourses.

\section{Literature review}

\subsection{Previous Studies of Metaphor}

The study of metaphor can be traced back to the ancient Greek period, when Aristotle considered metaphor a figure of speech for decorative use in literature and rhetoric. In his work Rhetorics and Poetics (1954), Aristotle argues that metaphor is a comparison between two things with shared similarity and resemblance. Quintilan put forward substitution theory based on comparison theory, claiming that metaphor is a phenomenon where one word is used to substitute another (Shu Ding Fang, 2002). All these two theories regard metaphor as a rhetorical device for ornamental use. After that, Richards proposed interaction theory, in which metaphor is an interaction of two concepts. This theory studies metaphor not from rhetorics, but semantics. In 1980, Lakoff and Johnson put forward conceptual metaphor theory in their work Metaphors We Live By, shifting metaphor research to a cognitive view. Different from the traditional view, conceptual metaphor regards metaphor as cognitive ability. Domestic study on metaphor from the perspective of cognitive linguistics began in the 1980s when Metaphors We Live By was introduced into China. Zhao Yanfang (1994) became the first in China to study the cognitive foundation of metaphor and made a book review on Metaphors We Live By (1995). In his work Studies in Metaphor, Shu Dingfang (2000) comprehensively discusses the causes, working mechanism, characteristics, and functions of conceptual metaphor. Hu Zhuanglin (2004), in his book Metaphor and Cognition summarises foreign metaphorical research.

\subsection{Previous Studies of Economic Discourse from the Perspective of Conceptual Metaphor}

Since Lakoff and Johnson proposed conceptual Metaphor Theory, it has been employed as a theoretical framework to study different discourses in different domains and economic discourse. Many foreign researchers have researched economic news from the perspective of cognitive linguistics (Henderson, 1982; McCloskey (1985); Krugman, 1997; Boers, 2000; CharterisBlack,2005). For example, Henderson (1982) was the first to study metaphors in economic discourse. McCloskey (1995) claims that metaphor is pervasive in economic discourse and metaphor is important for economic thinking. Besides, metaphor serves as a powerful cognitive device in writing economic news. Domestic scholars have also done much research on economic news from the perspective of cognitive linguistics (Kong, 2002; Li, 2005; Cui, 2013; Luo, 2018; Wang, 2018; Gao, 2019; Xuan, 2019). For example, Kong (2002) analyzes Chinese and German economic news and summarizes seven categories of conceptual metaphors. Cui (2013) identified six types of conceptual metaphors in the China Column of The Economist. Luo (2018) compares Chinese China-US strategic and Economic Dialogues with a conceptual metaphor approach. Wang (2018) makes a comparative study of conceptual metaphors in Chinese and American Economic News Headlines.

\subsection{Comments on Previous Studies}

The above analysis shows that through conceptual metaphors are widely employed in various discourses, including economic news, research on conceptual metaphors in The Economist is rare, particularly in Finance \& Economics Column. This is why this paper attempts to study conceptual metaphors in the Finance \& Economics Column of The Economist.

\section{Theoretical Framework}

\subsection{Definition of Conceptual Metaphors}

Conceptual Metaphor Theory was put forward by Lakoff and Johnson (1980) in their work Metaphors We Live By, which marks the beginning of studying metaphor from cognitive linguistics. They Held that metaphor is a pervasive phenomenon in daily life, not just in language but also in thought and action. Conceptual metaphor is a cognitive device, which allows people to understand abstract concepts with the help of concrete things. They consider metaphor as a cross-domain mapping in the conceptual system, mapping from the source domain to the target domain. Lakoff and Johnson concluded two essential conceptual metaphor characteristics: systematicity and cultural coherence (1980). Systematicity that allows us to comprehend one aspect of a concept in terms of another will hide other aspects of the concept (Lakoff \& Johnson,2003: 10). Cultural coherence means metaphors are not only coherent in themselves but also make their metaphorical expressions coherent.

\subsection{Classification of Conceptual Metaphors}

Lakoff and John divided conceptual metaphors into three categories: structural, ontological, and orientational metaphors (Lakoff \& Johnson, 1980). Structural metaphor means that "one concept is metaphorically structured in terms of another. It allows us to map familiar concepts onto unfamiliar and abstract concepts. For example, ARGUMENT IS WAR. The target domain 
"ARGUMENT" is structured in terms of the source domain "WAR". Ontological metaphor can be defined as "understanding human experiences in terms of objects and substances". In other words, we can project obscure concepts such as events, idea etc., onto entities and substances to quantify them, refer to them, and categorize them. A typical example of ontological metaphor is INFLATION IS AN ENTITY: Inflation is lowering our living standard. In this example, inflation is conceptualized as an entity, so that it can be quantified as an entity. Lakoff and Johnson defined orientational metaphor as "organizing a whole system of concepts with respect to another. For example, HAPPY IS UP metaphor. If we are happy, we usually say I'm feeling up to express our happy mood. Most orientational metaphor are relevant to spatial orientation.

\subsection{Working Mechanism of Conceptual Metaphors}

According to Lakoff and Johnson, the working mechanism is a kind of cross-domain mapping from the source domain to the target domain. It is a partial, asymmetric mapping process. That is to say, not all source domain features can be processed onto the target domain. The source domain is what we are familiar with, while the target domain is what we are unfamiliar with.

Take LIFE IS A JOURNEY as an example. LIFE is obviously the obscure concept that people use the source domain JOURNEY to help understand. The source domain "JOURNEY" is mapped onto the target domain "LIFE". The mapping process is showed as follows:

\begin{tabular}{|l|l|}
\hline Source domain & Target domain \\
\hline JOURNEY & LIFE \\
\hline Starting point & Birth \\
\hline Traveler & Person \\
\hline Path & Aging \\
\hline Crossroads & Choices \\
\hline Obstacles & Problems in life \\
\hline Destination & Death \\
\hline
\end{tabular}

Table 1 Mapping process of LOVE IS A JOURNEY Metaphor

According to Table 1, JOURNEY is the familiar source domain and LIFE is the abstract target domain. The elements of the source domain are mapped onto those of the target domain.

\section{Research Methods}

\subsection{Research Questions}

The paper is to analyze the economic news in the Finance \& Economics Column of the Economist from the perspective of conceptual metaphor. It tries to answer the following research questions.

(1) What types of conceptual metaphors are employed in the Finance \& Economics Column of the Economist?

(2) How do these types of conceptual metaphors mapping from the source domain to target domain?

(3) Why are these types of conceptual metaphors employed differently in the Finance \& Economics Column of the Economist?

\subsection{Data Collection}

This paper is to study the Finance \& Economics Column of the Economist from the perspective of conceptual metaphor, so the data were selected from the Finance \& Economics Column. Timespan is from October 2019 to December 2019, with 13 volumes. The size of the self-constructed corpus is 45000 words in total.

\subsection{Analytical Procedures}

After collecting the data, the corpus tool, AntConc is used to retrieved metaphorical keywords in the corpus. After that, the author needs to identify whether keywords retrieved by AntConc are metaphorical or not. They are identified in accordance with the criteria of Lakoff and Johnson's conceptual metaphor. And then, the sentences, including metaphorical keywords, are 
analyzed by the author. The mapping process from the source domain to the target domain is also presented. This study adopts a mixed research method, that is, qualitative method and quantitative method. The qualitative method is employed to analyze the use of conceptual metaphors. The quantitative method is used to count the frequency of conceptual metaphor of each category.

\section{Data Analysis and Discussion}

\subsection{Conceptual Metaphors in Finance \&Economics Column of the Economist}

According to Lakoff and Johnson (1980), metaphors are pervasive in our daily life, so as in economic news. With the help of corpus tools, AntConc, altogether $\mathbf{4 4 3}$ metaphors in self-constructed corpus are identified. And after analyzing, three kinds of conceptual metaphors classified by Lakoff and Johnson (1980) are all employed in the self-built corpus. Due to space limitations, only three major metaphors are selected to be explored in detail: the JOURNEY metaphor. HUMAN BEING metaphor and UPDOWN metaphor. All these identified conceptual metaphors are important for readers to understand obscure economic terminologies or events. The frequencies of different categories of conceptual metaphors identified in the chosen news are presented in Table 2.

\begin{tabular}{lcl}
\hline Conceptual Metaphor & Categories & Frequency \\
\hline Structural Metaphor & JOURNEY & 185 \\
\hline Ontological Metaphor & HUMAN BEING & 86 \\
\hline Orientational Metaphor & UP/DOWN & 172 \\
\hline
\end{tabular}

Table 2 Frequency of Conceptual Metaphors

From the above table, it can be seen that structural metaphors are most used, appearing 185 times. The number of ontological metaphors is 86. Orientational metaphors appear 172 times. The ontological metaphors are used less frequently than orientational metaphors, but the concrete types of orientational metaphors are fewer than ontological ones. Table 3 provides the Frequency of all concrete categories of metaphor in the self-constructed corpus.

\begin{tabular}{lll}
\hline Conceptual Metaphor & Categories & Percentage \\
\hline Structural Metaphor & JOURNEY & $42.04 \%$ \\
\hline Ontological Metaphor & LIVING ORGANISM & $19.54 \%$ \\
\hline Orientational Metaphor & UP/DOWN & $39.09 \%$ \\
\hline
\end{tabular}

Table 3 The Frequency of all Concrete Categories of Conceptual Metaphor

It can be found from Table 2 that the Frequency of JOURNEY metaphor is on the top with $42.04 \%$ of the total. UP/DOWN metaphor ranks the second place with a high frequency of $39.09 \%$. Following is HUMAN BEING metaphor with a frequency of $19.54 \%$ of the total number.

\subsubsection{Structural Metaphors in Finance \& Economics Column of The Economist}

Lakoff and Johnson (1980) defined structural metaphor as "one concept is metaphorically structured in terms of another. The JOURNEY metaphor, WAR metaphor, BUILDING metaphor, and GAME metaphor are all identified in the corpus, but only JOURNEY metaphor is analyzed in detail due to space limitation.

\subsubsection{JOURNEY Metaphor}

In the journey process, there are a starting point, traveler, path, destination, obstacles and crossroads. In the self-built corpus, JOURNEY metaphor is used to describe economic growth. In the following parts, these target domains are presented in accordance to the source domain, for example, FORWARD MOVEMENT Metaphor, BACKWARD MOVEMENT Metaphor, NO MOVEMENT Metaphor, SPEED Metaphor, OBSTACLES Metaphor, PATH Metaphor and DESTINATION Metaphor. Table 4 presents the metaphorical keywords and frequency of JOURNEY metaphor. 


\begin{tabular}{|c|c|c|c|}
\hline Metaphor & Metaphor & Keywords & Frequency \\
\hline \multirow{17}{*}{$\begin{array}{l}\text { JOURNEY } \\
\text { Metaphor }\end{array}$} & FORWARD MOVEMENT Metaphor & push & 16 \\
\hline & & Peak & 7 \\
\hline & & climb & 2 \\
\hline & & go further & 1 \\
\hline & BACKWARD MOVEMENT Metaphor & back & 32 \\
\hline & & fall & 29 \\
\hline & & return & 18 \\
\hline & NO MOVEMENT Metaphor & wait & 4 \\
\hline & & halt & 2 \\
\hline & SPEED Metaphor & rate & 43 \\
\hline & & fast & 9 \\
\hline & & pace & 5 \\
\hline & OBSTACLES Metaphor & obstacles & 1 \\
\hline & PATH Metaphor & road & 5 \\
\hline & & path & 2 \\
\hline & DESTINATION Metaphor & aim & 8 \\
\hline & & goal & 1 \\
\hline
\end{tabular}

Table 4 Metaphorical Keywords and Frequency of JOURNEY Metaphor

During a journey, travels may move forward, turn back or standstill. There are some similarities between economic development and a journey. The economic activities can make progress, retrogress, or come to a halt due to some reasons. Moving forward in a journey usually means the traveller is getting closer to the destination. By mapping into economic activities, it means economic activities have made an achievement.

From Table 4, it can be seen that "push", "peak" and "climb" are the four keywords employed in the FORWARD MOVEMENT metaphor to refer to the progressing in economic activities. "push" appears most frequently, following is "peak" with 7 times." "climb" and "go furthest" is employed less frequently. The following sentences are some typical examples selected from the corpus.

(1) It is still $60 \%$ below its 2007 peak.

(October $5^{\text {th }}$ 2019)

(2) France has gone furthest, with a 3\% levy on sales that will be backdated to the start of 2019. Britain's version, levying 2\%, is set to kick in next April.

(December $7^{\text {th }}$ 2019) 
In these two examples, moving forward in the process of a journey is mapped onto the progress made in economic activities. the target domain is conceptualized as the source domain; that is, the development of economic activities is conceptualized as a journey based on the structural metaphor: "DEVELOPMENT IS A JOURNEY".

In example (1), "peak" is the metaphorical keyword. In the source domain, it means the peak of a mountain. In this metaphor, "peak" refers to the economic development in Greece striking a new high. In the mapping process from source domain to target domain, economy in Greece is conceptualized as the traveler in Journey. The development of economy in Greece corresponds to traveler in a journey getting closer to the destination. This metaphor illustrates that Greece's economic development strikes a new height in 2007.

In example (2), the metaphorical keyword "go furthest" in the source domain means the traveler getting closer to the destination. In this metaphor, "furthest" refer to the increase in tax rates. Based on "DEVELOPMENT IS A JOURNEY", the traveler in a journey is mapped onto tax rate in France. The increase in tax rates is conceptualized as traveler moving forward. This metaphor illustrates that tax rates in France increasing to a new height.

In contrast, backward movement, most of the time, means traveler is far from the destination. Based on "DEVELOPMENT IS A JOURNEY", backward movement is conceptualized as declining economic activities. No movement metaphor means traveler stops for rest or is attracted by beautiful landscape, so there is no movement in the journey. In the target domain, economic activities may also stop for some reasons.

According to Table 4, there are three metaphorical keywords "back", "fall', "return" used in the backward movement to refer to the declining of economic activities. "back" appears most frequently, following is "fall" with 29 times." "return" appears 18 times. "wait' and "halt" identified in the selected news are employed In no movement metaphor. "wait" appears 4 times, while "halt" appears less frequently, with only two times. The following sentences are some typical examples selected from the corpus.

(3) The upshot, Mr Cai calculates, is that China's potential growth rate has fallen to about 6.2\%-almost exactly where it is today. (November $2^{\text {nd }}$ 2019)

(4) As the Democrats' impeachment inquiry gains steam, and business is halted, the prospects of another round of stimulus look dim.

(October 12 ${ }^{\text {th }}$ 2019)

In example (3), the metaphorical keyword "fallen" in the source domain means the traveler getting far from the destination. In this metaphor, "fallen" refer to the decrease of the growth rate in China. Based on "DEVELOPMENT IS A JOURNEY", the traveler in a journey is mapped onto the economic growth rate in China. The reduction in growth rates is conceptualized as travelers moving backwards.

Example (4) is based on "BUSINESS DEVELOPMENT IS A JOURNEY". The metaphorical keyword "halt" in the source domain means the traveler stops for some factors. In the target domain, it means the suspension of business activities. In this metaphor, the traveler in a journey is mapped onto business development. The suspension of business activities is conceptualized as traveler making no movement.

In a journey, speed is an important factor for travelers when heading toward the destination. Rapid speed will enable travelers to arrive at their destination in advance. On the contrary, if too slow, travelers may fail to arrive in time. In terms of economic news, the speeds of economic development or other economic activities are conceptualized as the speed of journey, so the speeds of economic development or other economic activities are interpreted in terms of the speed of journey.

The keywords of SPEED metaphor are listed in Table 4 with their frequencies. It can be seen that there are three keywords identified in the SPEED metaphor. Among them, the metaphorical keyword "rate" appears most frequently, following is "fast", with 9 times. "race" is used 5 times. The following sentences are some examples taken from the corpus.

(5) The upshot, Mr Cai calculates, is that China's potential growth rate has fallen to about 6.2\%-almost exactly where it is today. (October $12^{\text {th }}$ 2019)

(6) A new government aspires to get the economy growing fast. (October $5^{\text {th }}$ 2019)

Example (5) is based on "CHINA'S ECONOMIC GROWTH IS A JOURNEY.", in which the target domain "China's economic growth" is conceptualized as the source domain "the speed of journey", so we can understand China's economic growth with the help of our experience of the journey. China's economic growth corresponds to the speed of a journey. 
In example (6), the metaphorical keyword "fast" in the source domain means travellers travel at a rapid speed. In this metaphor, "fast" refers to the economy growing rapidly. In the mapping process from source domain to the target domain, the speed of economic growth is conceptualized as the speed of a journey, so the sentence "A new government aspires to get the economy growing fast" can be understood in terms of our life experience.

Besides, during a journey, travellers may come across some obstacles, which may derail the advancements when heading toward the destination. The OBSTACLE metaphor in economic news refers to a series of difficulties in economic activities. The difficulties in the process of a journey are mapped onto the obstacles such as inflation in economic growth or other economic activities. As shown in Table 4, we can see that only one word, "obstacles" is identified in the self-constructed corpus. The following sentence is an example selected from the corpus.

(7) When insurers opened for business in other South-East Asian countries ten to 20 years ago, they encountered, and surmounted, similar obstacles.

(December $7^{\text {th }}$ 2019)

Example (7) is based on "BUSINESS DEVELOPMENT IS A JOURNEY" in which the target domain "obstacles in starting a business" is conceptualized as the source domain "setbacks during a journey". In the mapping process from the source domain to target domain, setbacks during a journey is mapped onto obstacles in starting a business, so the sentence "When insurers opened for business in other South-East Asian countries ten to 20 years ago, they encountered, and surmounted, similar obstacles" can be understood in terms of our traveling experience.

In a journey, the traveler must choose a road or path when heading toward a destination. When starting an economic activity, a road or a path must be decided to achieve accomplishment. By mapping process, the source domain "path in a journey" is mapped onto the target domain "path in economic activities". As is shown in Table 4, there are two metaphorical keywords "road", with 5 times and "path" with 2 times. Here are some examples identified in the economic news.

(8) The road ahead will not be easy for Chinese exporters.

(December $14^{\text {th }}$ 2019)

(9) Across the rich world, forward guidance on the path of interest rates has become part of the toolkit.

(December $14^{\text {th }}$ 2019)

Example (8) is based on "TRADE DEVELOPMENT IS A JOURNEY". "road" in the source domain means the way the traveller takes when heading toward the destination. This metaphor refers to the way how China exports products to other countries. The target domain "road in China's trade development" is conceptualized as the source domain "path in a journey". "road in China's export" is consistent with "road in a journey", which enables readers to understand economic news more easily.

Example 9 is based on "the changing of interest rates is a journey", in which the target domain "path of interest rates" is conceptualized as the source domain "path during a journey". Therefore, we can understand the changing of interest rates in bank in terms of our traveling experience. After the mapping process, the path in a journey can be used to talk about how interest rates change.

Finally, in the process of a journey, the traveler will arrive at the destination. The DESTINATION metaphor in economic news refers to the goal of economic activities. The aim of economic activity is conceptualized as the destination in a journey. From Table 4, it can be seen that two metaphorical keywords "aim" and "goal" used in DESTINATION metaphor. "aim" appears eight times, while "goal" appears only one time. The following two sentences are some examples taken from the corpus.

(10) If interest rates are cut further in pursuit of that goal, the country risks another currency crisis. (November $2^{\text {nd }}$ 2019)

(11) In the hope of reviving inflation, the ecb has pledged to keep rates low and continue buying bonds until underlying inflation returns to its aim of "close to, but below, $2 \%$ ".

(October 12 ${ }^{\text {th }}$ 2019)

In example (10), the metaphorical keyword "goal" in the source domain means the destination that travellers arrive at. In this metaphor, "goal" means the goal of the trade between China and US. In the mapping process from source domain to the target domain, the trade goal between China and the US is conceptualised as a journey's destination.

Example (10) is based on "INFLATION CHANGING IS A JOURNEY". The metaphorical keyword "aim" in the source domain means the destination that travelers arrive at. By mapping process, the target domain "the aim of curtailing inflation" is 
conceptualized as the source domain "the destination of a journey". Therefore, the sentence "In the hope of reviving inflation, the ecb has pledged to keep rates low and continue buying bonds until underlying inflation returns to its aim of "close to, but below, $2 \% "$ can be understood in terms of journey destination.

The source domain "journey" can be mapped onto the target domain "economic activities" from the above analysis. Conceptual metaphors include DEVELPOMENT IS A JOURNEY, ECONOMIC GROWTH IS A JOURNEY, INFLATION CHANING IS A JOURNEY. The following figure shows us the mapping process of the JOURNEY metaphor, which can help us understand the target domain in terms of the source domain.

\begin{tabular}{|ll|}
\hline \multicolumn{1}{|c|}{ JOURNEY } & $\begin{array}{c}\text { ECONOMIC DEVELOPMENT } \\
\text { (Source domain) }\end{array}$ \\
DISPLACEMENT $\longrightarrow$ Darget domain $)$ \\
PATH $\longrightarrow$ DEVELOPMENT TRACKS \\
SPEED $\longrightarrow$ DEVELOPMENT SPEED \\
OBSTACLE $\longrightarrow$ DIFFIVULTY
\end{tabular}

Figure 1 Metaphorical mapping process of JOURNEY metaphor

\subsubsection{Ontological Metaphors in Finance \& Economics Column of The Economist}

According to Lakoff and Johnson (1980), ontological metaphor can be defined as "understanding human experiences in terms of objects and substances". The study only selects HUMAN BEING metaphor to analyze.

\subsubsection{HUMAN BEING Metaphor}

People tend to understand some abstract concepts in terms of human features in daily life, such as human feelings and the human body. In the selected economic news, human features are often attributed to economic terms to help readers understand more easily; that is, the economy can be understood as a person. Table 5 presents the metaphorical keywords and frequency of HUMAN BEING metaphor.

\begin{tabular}{|c|c|c|c|}
\hline Metaphor & Metaphor & Keywords & Frequency \\
\hline & & growth & 43 \\
\hline & LIFE CIRCLE & grow & 12 \\
\hline & & thrive & 2 \\
\hline \multirow[t]{6}{*}{ HUMAN BEING } & & infancy & 1 \\
\hline & THE BODY PART & arm & 1 \\
\hline & & brain & 4 \\
\hline & & suffer & 12 \\
\hline & HEALTH & hurt & 5 \\
\hline & & recover & 5 \\
\hline
\end{tabular}


paralysed

Table 5 Metaphorical Keywords and Frequency of HUMAN BEING Metaphor

As shown in table 5, there are three kinds of metaphors in the HUMAN BEING metaphor in the self-built corpus: LIFE CIRCLE metaphor, THE BODY PART metaphor, and the HEALTH metaphor. LIFE CIRCLE metaphor appears most frequently, the following is HEALTH metaphor. THE BODY PART metaphor is employed less frequently. Within LIFE CIRCLE metaphor, "growth" appears most frequently, with 12 times. Within HEALTH metaphor, "suffer" is used most frequently, with 12 times. Within THE BODY PART metaphor, "brain" appears 5 times. The following sentences are some examples from the self-constructed corpus.

(12) But for now the ESG rating industry is still in its infancy and Ms Peirce's criticisms, though blunt, ring true.

(December $7^{\text {th }}$ 2019)

(13) But annual GDP growth rates have been anaemic, at 1-2\%.

(October $5^{\text {th }}$ 2019)

(14) It is also because economic growth has slowed, with oil demand suffering not just in Japan but in India and South-East Asia, where it was expected to grow strongly.

(November $16^{\text {th }} 2019$ )

In the above examples, "infancy", "growth", "grow" are the metaphorical keywords employed in LIFE CIRCLE metaphor. The economy, like children, also experiences its infancy stage. Example (12) is based on INDUSTRY is HUMAN BEING. In this metaphor, the target domain "the infancy stage of ESG rating industry" is conceptualized as the source domain "children's infancy. By mapping process, readers is easy to understand that the ESG rating industry is still in its primary stage. Example (13) (14) are based on ECONOMY IS HUMAN BEING. "growth" and Its noun "growth" are metaphorically used to describe the improvement of some economic activities, such as GDP, productivity, revenue and so on. In the mapping process from the source domain to the target domain, the source domain "human growth" is mapped onto "economy growth".

(15) That is dismal news for a company already suffering from brain drain.

(November $5^{\text {th }}$ 2019)

In example (15), the metaphorical word "brain" in the source domain is an important part of human beings. In the target domain, it may refer to the capability of the company in economic activities. It is based on "COMPANAY IS HUMAN BEING". By mapping process, the target domain "company" is mapped onto the source domain "human being".

(16) The currencies of other small countries with substantial foreign trade had also suffered. (December 14th 2019)

(17) How the twists and turns of the trade war are hurting growth.

(October 19th 2019)

(18) Global revenues are at least recovering from a low of just \$14.3bn in 2014, when piracy was rampant and business models had yet to adapt to the digital age.

(October $5^{\text {th }}$ 2019)

(19) Mr Mitsotakis must deal with twin legacies of the crisis: paralysed banks and cripplingly tight fiscal policy.

(October $5^{\text {th }}$ 2019)

In the above examples, "suffered", "growth", "recovering" and "paralysed" are four metaphorical keywords employed in HEALTH metaphor. Health condition is a problem human beings must face. Based on HEALTH metaphor, health problems are often attributed to describe economic activities. Example (16) is based on "FOREIGN TRADE IS HUMAN BEING". Like a human being, foreign trade also encounters certain hinderance and suffers from it. Example (17) is based on "TRADE WAR IS ILLNESS". Trade war, like illness, also hurts economic growth. Example (18) is based on "ECONOMIC CRISIS IS ILLNESS". In this metaphor, global revenues suffered from a crisis in 2014. After a long period of time, now they recover from the disease condition.

From the above analysis, the source domain "HUMAN BEING" is used to describe the conditions of economic activities. Conceptual metaphors include "INDUSTRY is HUMAN BEING", "ECONOMY IS HUMAN BEING", "TRADE WAR IS ILLNESS", "FOREIGN TRADE IS HUMAN BEING", "ECONOMIC CRISIS IS ILLNESS". The source domain "HUMAN BEING" is mapped onto the target domain "ECONOMY". The mapping process of the HUMAN BEING metaphor is shown in the following figure 2. 


\begin{tabular}{|lll|}
\hline HUMAN BEING & $\begin{array}{l}\text { ECONOMY } \\
\text { (Source domain) }\end{array}$ \\
LIFE CIRCLE & $\begin{array}{l}\text { (Target domain) } \\
\text { THE BODY PART }\end{array}$ & DEVELOPMENT STAGE \\
HEALTH & STRUCTURE \\
& & DEVELOPMENT CONDITION \\
\hline
\end{tabular}

Figure 2 Metaphorical mapping process of JOURNEY metaphor

\subsubsection{Orientational Metaphors in Finance \& Economics Column of The Economist}

Lakoff and Johnson (1980) defined orientational metaphor as "organizing a whole system of concepts with respect to another. The study only selects UP/DOWN metaphor to analyze.

\subsubsection{UP/DOWN Metaphor}

In the self-constructed corpus, UP/DOWN metaphor is employed most frequently among orientational metaphors. They are mostly employed to describe the fluctuation of prices, production, interest rates, payment, etc. The UP/DOWN metaphor identified in the selected economic news can be divided into three types: MORE IS UP; LESS IS DOWN. Table 6 shows the metaphorical keywords and frequency of UP/DOWN metaphor.

\begin{tabular}{|c|c|c|}
\hline Metaphors & Keywords & Frequency \\
\hline \multirow{9}{*}{ MORE IS UP } & rise & 25 \\
\hline & raise & 20 \\
\hline & up & 16 \\
\hline & increase & 15 \\
\hline & boost & 6 \\
\hline & push up & 5 \\
\hline & prop up & 4 \\
\hline & boom & 4 \\
\hline & quadruple & 1 \\
\hline \multirow{5}{*}{ LESS IS DOWN } & cut & 32 \\
\hline & fall & 21 \\
\hline & drop & 7 \\
\hline & down & 6 \\
\hline & decline & 6 \\
\hline
\end{tabular}


slump

4

Table 6 Metaphorical keywords and frequency of UP/DOWN metaphor

As shown in table 6, MORE IS UP metaphor is employed more frequently than LESS IS DOWN metaphor in the current corpus. Within MORE IS UP metaphor, "rise" appears most frequently, with 25 times, while "cut" in LESS IS DOWN metaphor appears 32 times. The following sentences are several examples selected from the corpus.

(20) It could have been worse - the Americans could have applied a tariff of up to 100\%-but even one of $10 \%$ will bite. American airlines fear that tariffs will raise the price of Airbus aircraft, and that Boeing will lack the capacity to expand supply.

(October $5^{\text {th }}$ 2019)

(21) Pressing firms to raise pay, perhaps with faster increases in the minimum wage, could help in the short run but accelerate automation over the medium to long term.

(December $7^{\text {th }}$ 2019)

(22) The lira has partially recovered from a battering that had pushed domestic prices up. (November $2^{\text {nd }} 2019$ )

In the above examples, they are all based on MORE IS UP. In example (20), the increase of tariff can be regarded as an upward movement. Based on the physical basis of the MORE IS UP metaphor, the metaphorical keyword "up" can be used to understand the tariff increase. For example, (22), raising pay means more in wages. Based on MORE IS UP metaphor, it allows us to talk about the increase of wage. In example (22), when domestic prices increase, people have to spend more to buy products, so "pushing domestic prices up" can be understood in terms of MORE IS UP metaphor.

(23) Private employers added a net 358,000 jobs in the third quarter, down from 527,000 during the same period a year earlier, but still well in positive territory.

(October $5^{\text {th }}$ 2019)

(24) In July 2009, in the depths of the financial crisis, it was the first central bank to cut interest rates below zero.

(December $14^{\text {th }}$ 2019)

Above examples are all based on LESS IS DOWN metaphor. In example (23), the metaphorical word "down" is used to describe that employers are become less, "down" from 527000 to 358000. In example (24), based on LESS IN DOWN metaphor, the decrease of interest rates can be understood in terms of metaphorical keyword "cut", which can better help readers understand the actions that the central bank takes.

\subsection{Discussion of Differences of Conceptual Metaphors in Economic News}

From the above analysis, we can see that conceptual metaphor are employed differently in the corpus. The following discussions explore why different kinds of conceptual metaphors appear at different times from the perspective of cultural coherence and systematicity of conceptual metaphors.

\subsubsection{Differences of Conceptual Metaphors in Terms of Cultural Coherence}

According to Lakoff and Johnson (1980), conceptual metaphors are deeply connected with culture. Culture is different from country to country. Therefore, metaphors that are chosen to be employed in economic news vary from culture to culture. Language comes from particular cultural context, so language reflects the culture and is shaped by culture. The Economist is a typical financial journal in England, so the language used in The Economist must carry an English feature. Specifically, the conceptual metaphors employed in Finance \& Economics Column are mostly based on English culture.

Take UP/DOWN metaphor as an example; British people often consider "up" as "more" or "good". As a result, LESS IS UP metaphor is rarely employed to facilitate reader's understanding. Instead, "up" is often used to describe the progress made in economic activity while "down" is prone to describe the decline of economic activities. UP/DOWN metaphor is closely related to spatial environment. Some obscure concepts in the target domain are understood in terms of concepts or objects in the source domain closely connected with people's daily lives in structural and ontological metaphor. Therefore, Compared with WAR metaphor, BUILDING metaphor and MACHINE metaphor, JOURNEY metaphor and HUMAN BEING metaphor employed more frequently than other metaphors. 


\subsubsection{Differences of Conceptual Metaphors in Terms of Systematicity}

According to Lakoff and Johnson (1980), "Systematicity that allows us to understand one aspect of a concept in terms of another will unavoidably highlight certain aspects of one concept but hide other aspects of the concept. In other words, due to systematicity, some elements are prior to be chosen than others when conceptualizing one concept in terms of another. That is why we focus on certain aspects of a concept instead of other aspects of the concept that are not consistent with that metaphor. For instance, in TIME IS MONEY metaphor, not all elements of money are mapped onto time. For example, when you spend money to buy something, you can earn your money back, but you can earn your time back if you spend your time doing something.

Analyzing three kinds of conceptual metaphors, the impact of systematicity is pervasive throughout the economic news. In JOURNEY metaphor, some features in the source domain "JOURNEY", like forward, backward, no movement, path, obstacle, speed and destination, can be processed onto the target domain "economy" while some features cannot be processed. For instance, a beautiful landscape in the process of the journey cannot be mapped onto the target domain economy.

\subsubsection{Differences of Conceptual Metaphors in Terms of Characteristics of Economic Discourses}

Three kinds of conceptual metaphors analyzed in detail are employed disproportionately in the self-constructed corpus. The structural metaphor appears most frequently. Employed in economic news, structural metaphor facilitates comprehension by allowing readers to understand the concept in terms of others that are more familiar to readers. There are more metaphorical keywords identified in structural metaphor than another two metaphors. All identified metaphors of JOURNEY metaphor in the corpus are consistent with the characteristics of economic discourses.

The Economist is a kind of financial journal with an audience of native British, so there are plenty of obscure economic terminologies in the selected news discourse. Some abstract concepts in economy can be conceptualized as concrete source domain in ontological metaphor by employing an ontological metaphor. This may be why the HUMAN BEING metaphor is employed most frequently in the selected corpus in ontological metaphor.

The orientational metaphor employed most frequently is UP/DOWN metaphor. UP metaphor is used to describe the increase of price, production etc. On the contrary, DOWN metaphor is used to describe the decrease in price, production etc. It implicates the conciseness of economic news.

\section{Conclusion}

In conclusion, with a corpus-based approach, this paper applies Conceptual Metaphor Theory to analyze the economic discourses in the Finance \& Economics Column of The Economist and comes to the following conclusions. Firstly, a total of 443 conceptual metaphors are identified in the self-constructed corpus, which verifies the pervasiveness of conceptual metaphors in daily life, including economic discourses. Secondly, all three types of conceptual metaphors are employed in the corpus. However, due to space limitations, the paper only selects the most frequent one in each type, namely, JOURNEY, HUMAN BEING, and UP/DOWN. Conceptual metaphors used in economic news allow readers to map the source domain to the target domain to understand some abstract concepts. For example, economic development can be understood metaphorically as a journey process. Finally, the frequency of these three types of conceptual metaphor varies from each other. JOURNEY metaphors appear with $42.04 \%$, HUMAN BEING metaphors appear with $19.54 \%$ and UP/DOWN metaphors appear with $39.09 \%$, which indicates that culture may be a factor behind the frequency, since The economist is a British journal. Therefore, this paper expands the study scope of conceptual metaphor in the Finance \& Economics Column of The Economist by analyzing three types of conceptual metaphors and illustrates their working mechanism and why their frequency varies. Despite the theoretical and practical significance provided, this paper still has its limitations. Due to the space limitation, although many categories of conceptual metaphor have been identified in the corpus, such as WAR metaphor, BUILDING metaphor, MACHINE metaphor, the paper only analyze the most frequent metaphor in each category, namely, JOURNEY metaphor, HUMAN BEING metaphor, UP/DOWN metaphor, so it is not comprehensive. Secondly, the data is based on a small number of corpora, only covering three months of articles. Apart from retrieving process with the help of AntConc, the process of metaphor identification and analysis is all manual, so it unavoidably lacks subjectivity. And metaphor identification is not exhaustive. Despite the limitations listed above, this paper still offers some inspirations for future research and it is suggested that future studies in conceptual metaphor should explore many other domains or even connect with other disciplines. 


\section{Reference}

[1] Aristotle. (1954). Rhetoric and Phoetics. New York, NY: The Modern Library.

[2] Boers, F. (2000). Enhancing Metaphoric Awareness in Specialized Reading. English for Specific Purpose. 19(2), 137-147.

[3] Charteris-Black \& Jonathan. (2005). Politicians and Rhetoric: The persuasive power of metaphor. New York, NY: Palgrave MacMillan.

[4] Cui, Y. (2013). Jing ji yu pian zhong de gai nian yin yu_yi <Jing ji xue ren > zhong guo zhuan lan wei li [Conceptual Metaphor in Economic Discourses: A Case Study of Special Column China in The Economist]. Journal of Kunming Metallurgy College, 29(04), 76-80.

[5] Gao, X. (2019). A Corpus-based Study on Conceptual Metaphors in China Column of The Economist. Master Dissertation, Lanzhou University of Technology. China National Knowledge Infrastructure.

[6] Henderson, W. (1982). Metaphor in Economics. Economics. 18(4), 147-153.

[7] Hu, Z. (2004). Ren zhi yin yu xue [Metaphor and Cognition]. Beijing: Peking University Press.

[8] Kong, D. (2002). Cong ren zhi kan jing ji yu pian zhong de gai nian yin yu [Application of Metaphorical Concept in Economic News Reports]. Foreign Languages and Their Teaching, (02), 13-16.

[9] Krugman, P. (1997). Development, Geography and Economic Theory. London: The MIT Press.

[10] Lakoff. G \& Johnson. M. (1980). Metaphors We Live By. Chicago: University of Chicago Press.

[11] Lakoff. G \& Johnson. (2003). Metaphors we live by. London: The university of Chicago press.

[12] Mc Closkey, N. (1985). The Rhetoric of Economics. Madison: University of Wisconsin Press.

[13] Li, M. (2005). Shang wu yong yu zhong jing ji lei wen ben li de yin yu ji zhi ji gongneng [The Mechanism and Function of metaphor in economic texts in Business English]. Journal of Guangdong University of Foreign Studies, 16(02), 25-28.

[14] Luo, X. (2019). A Cognitive Approach to the Study of Conceptual Metaphors in Chinese China- U.S. Strategic and Economic Dialogues. Master Dissertation, Henan University. China National Knowledge Infrastructure.

[15] Su, D. (2000). Yin yu xue yan jiu [Studies in Metaphor]. Shanghai: Shanghai Foreign Language Education Press.

[16] Wang, J. (2018). Comparative Study of Conceptual Metaphors in Chinese and American Economic News Headlines from Cross-Cultural Communication Perspective. [Master Dissertation] Tianjing University of Commerce. China National Knowledge Infrastructure.

[17] Xuan, X. (2019). A Study of the Coherent Function of Conceptual Metaphors in English Economic News Discourses. Master Dissertation, Northeast Normal University. China National Knowledge Infrastructure.

[18] Zhao, Yan. (1994). Yin yu de ren zhi ji chu [The Cognitive Base of Metaphor]. Journal of PLA University of Foreign Languages, (02), 30-34. 